\title{
Cohesive crack propagation using a boundary element formulation with a tangent operator
}

\author{
E. D. Leonel \& W. S. Venturini \\ Department of Structural Engineering, \\ São Carlos School of Engineering, University of São Paulo, Brazil
}

\begin{abstract}
In this work, a BEM formulation is proposed to analyse cohesive crack using the dual reciprocity method. The solution of the non-linear system of equations is based on the Newton-Raphson process for which the consistent tangent operator is derived. The cohesive crack model is the criterion adopted to describe crack growth particularly for quasi-brittle material problems. The solution is very stable and accurate and can be easily applied to solids that exhibit many crack or micro-cracks. Examples are shown to illustrate the applicability of the formulation.
\end{abstract}

Keywords: boundary elements, crack growth, cohesive crack model.

\section{Introduction}

The boundary element method (BEM) has already been recognized as an accurate and efficient numerical technique to deal appropriately with many problems in engineering. In particular, the method is recommended to analyse crack problems. For this kind of problems the dimensionality reduction of BEM is clear, as only boundary discretization is required. Internal points are needed only to approximate the crack line, but without requiring remeshing. Among the crack models the Hillerborg cohesive model is an idealization that represents well the dissipation process occurring in a small region ahead the crack tip [1]. The dissipation zone is approximated by a crack line since the process begins. This model is particularly recommended for the analysis of quasi-brittle materials.

The Dual Boundary Element Method (DBEM), proposed by Portela et al. [2], is nowadays the most used technique to model linear and non-linear fracture 
processes. The method is based on the use of singular and hyper-singular integral equations written for collocations along the crack line. This scheme allows writing four independent relations for each node defined on the crack line and avoids the division of the solid into sub-regions. Besides these works we want to cite some alternative procedures proposed by the senior author: Venturini [3] has used dipoles to enforce crack discontinuities governed by a cohesive model; Manzoli and Venturini [4] have modelled cracks by using strong discontinuities similar to the technique used for the enhanced finite element methods, and Leite and Venturini [5] adopting narrow sub-regions with rigidity going to zero for the simulation of cracks.

The solution techniques for the majority of the proposed works to analyse crack problems using BEM are based on iterative schemes that corrects the forces between the crack faces that satisfy the criterion adopted (see for instance Saleh and Aliabadi [6]). These processes are simple and the relevant matrices are also kept constant during the process. This kind of technique requires a large number of iterations to achieve the equilibrium for a single load increment. Moreover, for the cases of more complex pattern of cracks, for instance with a solid containing many micro-crack, this process can be either inaccurate or instable.

In this work, we are analysing quasi-brittle two-dimensional domains using the cohesive crack model. Two solution techniques are proposed: the classical procedure based on the corrections of the crack surface forces and a more elaborated scheme for which the consistent tangent operator has been derived. for the second procedure the system of equations is solved by using the NewtonRaphson method. Numerical examples are shown to compare the accuracy and efficiency of the two procedures.

\section{Cohesive crack model}

The cohesive crack models, in which the dissipation zone was reduced either to a line for $2 \mathrm{D}$ problems or to a surface for $3 \mathrm{D}$ problems, appropriate to quasi-brittle materials are due to Hillerborg et al. [1]. In this work a softening region just behind the crack tip is defined along which a relation between the crack surface tensile forces, or cohesive forces $f_{t}$, and the crack opening displacement $\Delta u$ is defined to govern the dissipative process. The cohesive forces disappear after a crack opening critical value, $\Delta u_{c}$. The crack starts opening when the cohesive forces reaches a tensile critical value $f_{t}^{c}$. For values of the tensile stresses less than $f_{t}^{c}$ the crack does not open. For values of the crack opening displacement larger than $\Delta u_{c}$ the cohesive force is zero.

Several relations between cohesive forces and crack opening displacement have already been tested. Three of them are often employed to carry out crack analysis for quasi-brittle materials: linear model; bi-linear model; and exponential model (a) linear model; b) bi-linear model; and c) exponential model (Figure 1). 

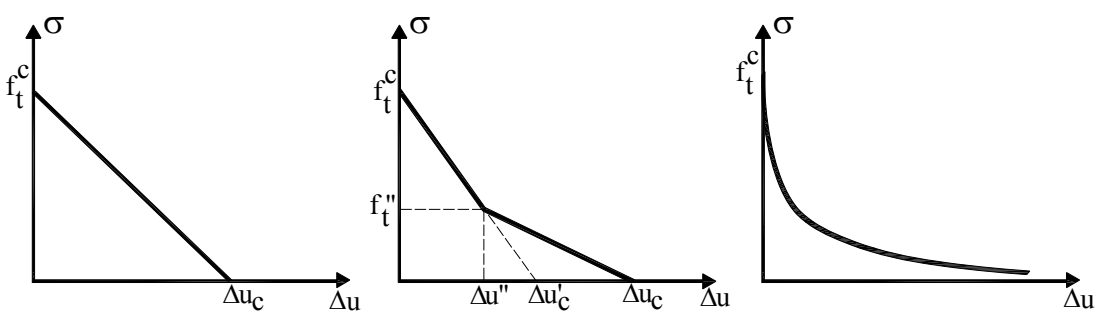

Figure 1: Cohesive force models. a) linear model ; b) bi-linear model; and c) exponential model.

For the linear case the cohesive model is given by the following relations:

$$
\begin{array}{cc}
\sigma=E \varepsilon & \text { if } \varepsilon \leq \varepsilon_{c} \\
f_{t}(\Delta u)=f_{t}^{c}\left(1-\Delta u / \Delta u_{c}\right) & \text { if } 0 \leq \Delta u \leq \Delta u_{c} \\
f_{t}(\Delta u)=0 & \text { if } \Delta u>\Delta u_{c r}
\end{array}
$$

\section{Dual Boundary Element Method}

For an elastic domain $\Omega$, with boundary $\Gamma$, one can easily derive the integral representation of displacements as follows:

$$
c_{i l}(f) u_{l}(f)+\int_{\Gamma} P_{i l}^{*}(f, c) u_{l}(c) d \Gamma=\int_{\Gamma} P_{l}(c) u_{i l}^{*}(f, c) d \Gamma
$$

where $u_{i j}^{*}$ and $p_{i j}^{*}$ are the Kelvin's fundamental solutions for displacement and tractions, $u_{j}$ and $p_{j}$ are boundary displacements and tractions and $c_{i l}$ the well known free term for elastic problems; it is equal to $\delta_{i l}$ for internal points, zero for outside points and $\delta_{i l} / 2$ for smooth boundary nodes and also nodes defined along the crack surfaces.

To obtain this integral representation one has to differentiate equation (6) to obtain the strain integral equation, apply the Hooke's law to achieve the stress integral representation and then multiply this equation by the director cosines of the crack surfaces to obtain the traction representation for smooth collocations as follows:

$$
\frac{1}{2} p_{j}(f)+\eta_{k} \int_{\Gamma} S_{k i j}(f, c) u_{k}(c) d \Gamma=\eta_{k} \int_{\Gamma} D_{k i j}(f, c) p_{k}(c) d \Gamma
$$

where the kernels $S_{k i j}$ and $D_{k i j}$ are derived from the traction and displacement fundamental values following the steps above described. 
Equations (2) and (3) are, as usual, transformed to algebraic relations by dividing the boundary and the crack surfaces into elements along which displacements and tractions are approximated. Besides that one has to select a convenient number of collocation points to obtain the algebraic representations. In this work we are using boundary collocation points either at the element ends, therefore coincident with nodes, or along the element when displacement and traction discontinuities are recommended. Using the discretized form of equation (2) applied only to boundary collocation points one can obtain the usual system of algebraic equations, relating boundary values, as follows:

$$
H_{b}^{b} U_{b}+H_{b}^{f} U_{f}=G_{b}^{b} P_{b}+G_{b}^{f} f
$$

where $U_{b}, U_{f}$, are displacements assigned to boundary (b) and to crack surface nodes $(f), P_{b}$ gives the boundary tractions, while $f$ represents the tractions applied along the crack surfaces; $H_{b}^{b}, H_{b}^{f}, G_{b}^{b}$ and $G_{b}^{f}$ are the corresponding matrices to take into account displacement and traction effects, the subscript $b$ indicates that the collocation is on the boundary and the superscripts specify the boundary $(b)$ or crack surface $(f)$ values.

For the crack surfaces we need two opposite collocations for each discretization node to obtain four algebraic independent relations, corresponding to four unknown crack surface values, two displacements and two tractions. As we are dealing with hyper-singular equations (3), it is convenient to use collocation points defined along the element and not coincident with the discretization node. The node values of crack displacements and tractions are however kept at the element end. Thus, from the discretized forms of equations (2) and (3), one can write the following set of algebraic equations:

$$
H_{f}^{b} U_{b}+H_{f}^{f} U_{f}=G_{f}^{b} P_{b}+G_{f}^{f} f
$$

where the subscript $f$ in the matrices $H_{f}^{x}$ and $G_{f}^{x}$ indicates equation written for collocation along the crack line.

Using equations (4) and (5) together with the cohesive crack model described in the previous section one can develop an appropriate algorithm to analyze crack problems as will be shown in the next section.

\section{Solution technique}

In the context of BEM a non-linear crack problem can be solved by simple schemes, in which the relevant matrices are kept constant and the corrections are applied to the crack surface forces according to the chosen criterion [6]. This technique is simple, but usually requires a large number of iterations to find the equilibrium within a load increment.

Non-linear implicit BEM formulations, employing tangent operators, have shown to give more accurate and stable results $[7,8]$. In what follows, this 
scheme is employed as an alternative technique to solve crack problems.

To derive the crack BEM approach using tangent operator let us modify the equilibrium equations (4) and (5) as follows

$$
\begin{aligned}
& H_{b}^{b} U_{b}+H_{b}^{r} U_{r}+H_{b}^{\ell} U_{\ell}=G_{b}^{b} P_{b}+G_{b}^{r} P_{r}+G_{b}^{\ell} P_{\ell} \\
& H_{f}^{b} U_{b}+H_{f}^{r} U_{r}+H_{f}^{\ell} U_{\ell}=G_{f}^{b} P_{b}+G_{f}^{r} P_{f}^{r}+G_{f}^{\ell} P_{\ell}
\end{aligned}
$$

where the subscripts $r$ and $\ell$ are related to the right and left crack surfaces.

Crack surface displacements and tractions in Equations (6) and (7) have to be modified to be given in local coordinates $(n, s)$, in which $n$ and $s$ are coordinate axes perpendicular and parallel to the crack line, respectively. Then, one can define the crack opening displacements $u_{s}$ and $u_{n}$ as follows

$$
U_{\ell s}=u_{s}-U_{r s} \quad U_{\ell n}=u_{n}-U_{r n}
$$

After these modifications equations (6) and (7) read

$$
\begin{aligned}
& H_{b}^{b} U_{b}+\left(H_{b}^{r s}-H_{b}^{\ell s}\right) U_{r s}+\left(H_{b}^{r n}-H_{b}^{\ell n}\right) U_{r n}+H_{b}^{\ell s} u_{s}+ \\
& H_{b}^{\ell n} u_{n}=G_{b}^{b} P_{b}+G_{b}^{r s} f_{r s}+G_{b}^{r n} f_{r n}+G_{b}^{\ell s} f_{\ell s}+G_{b}^{\ell n} f_{\ell n} \\
& H_{f}^{b} U_{b}+\left(H_{f}^{r s}-H_{f}^{\ell s}\right) U_{r s}+\left(H_{f}^{r n}-H_{f}^{\ell n}\right) U_{r n}+H_{f}^{\ell s} u_{s}+ \\
& H_{f}^{\ell n} u_{n}=G_{f}^{b} P_{b}+G_{f}^{r s} f_{r s}+G_{f}^{r n} f_{r n}+G_{f}^{\ell s} f_{\ell s}+G_{b}^{\ell n} f_{\ell n}
\end{aligned}
$$

Equations (9) and (10) have to be written and solved within the context of incremental problems. Thus, for a given increment of load one has to replace the boundary and crack values by the corresponding increments. Then, after applying the boundary conditions, as usual we have to store all unknown boundary increments into the vector $\Delta X$ and cumulate the known boundary value effects into the independent vectors $\Delta F_{b}$ and $\Delta F_{f}$. Thus, equations (9) and (10) become

$$
\begin{aligned}
& Y_{b}=A_{b} \Delta X+H_{b}^{\ell n} \Delta u_{n}-\Delta F_{b}-\left(G_{b}^{r n}-G_{b}^{\ell n}\right) \Delta f_{r n}=0 \\
& Y_{f}=A_{f} \Delta X+H_{f}^{\ell n} \Delta u_{n}-\Delta F_{f}-\left(G_{f}^{r n}-G_{f}^{\ell n}\right) \Delta f_{r n}=0
\end{aligned}
$$

where $A_{b}$ and $A_{f}$ contain the coefficients of matrices referred to unknown boundary and crack surface displacement increments $\left(\Delta U_{b}, \Delta U_{r s}\right.$ and $\left.\Delta U_{r n}\right)$, unknown boundary traction increments $\left(\Delta P_{b}\right)$, and the fictitious crack opening increments in the direction $s\left(\Delta u_{s}\right)$. The traction increments in the direction $s$ are neglected according to the adopted cohesive crack model. Moreover, the left 
surface crack tractions $\Delta f_{\ell n}$ are replaced by minus right surface crack tractions, $-\Delta f_{r n}$.

To find the solution of equations (11) and (12) one has to linearize these equations using only the first term of the Taylor's expansion, as follows

$$
\begin{aligned}
& Y_{b}\left(\Delta u_{n k}^{i}\right)+\frac{\partial Y_{b}\left(\Delta X_{k}^{i}, \Delta u_{n}^{i} \ldots\right)}{\partial \Delta X_{k}^{i}} \delta \Delta X_{k}^{i}+\frac{\partial Y_{b}\left(\Delta X_{k}^{i}, \Delta u_{n}^{i} \cdots\right)}{\partial \Delta u_{n k}^{i}} \delta \Delta u_{n k}^{i}=0 \\
& Y_{f}\left(\Delta u_{n k}^{i}\right)+\frac{\partial Y_{f}\left(\Delta X_{k}^{i}, \Delta u_{n}^{i} \cdots\right)}{\partial \Delta X_{k}^{i}} \delta \Delta X_{k}^{i}+\frac{\partial Y_{f}\left(\Delta X_{k}^{i}, \Delta u_{n}^{i} \ldots\right)}{\partial \Delta u_{n k}^{i}} \delta \Delta u_{n k}^{i}=0
\end{aligned}
$$

Carrying out all indicated derivatives in equations (13) and (14) the following consistent tangent matrix is derived,

$$
[C]=\left[\begin{array}{ll}
A_{b} & {\left[H_{b}^{\ell n}-\left(G_{b}^{r n}-G_{b}^{\ell n}\right) \partial \Delta f_{r n} / \partial \Delta u_{n k}^{i}\right]} \\
A_{f} & {\left[H_{f}^{\ell n}-\left(G_{f}^{r n}-G_{f}^{\ell n}\right) \partial \Delta f_{r n} / \partial \Delta u_{n k}^{i}\right]}
\end{array}\right]
$$

where the derivatives $\partial \Delta f_{r n} / \partial \Delta u_{n k}^{i}$ are obtained by differentiating properly the adopted cohesive crack law.

Thus, the corrections $\delta \Delta X_{k}^{i} \delta \Delta u_{n k}^{i}$, obtained from equations (13) and (14), is given by

$$
\left\{\begin{array}{c}
\delta \Delta X_{k}^{i} \\
\delta \Delta u_{n k}^{i}
\end{array}\right\}=-[C]^{-1}\left\{\begin{array}{c}
Y_{b}\left(\Delta u_{n k}^{i}\right) \\
Y_{f}\left(\Delta u_{n k}^{i}\right)
\end{array}\right\}
$$

Within a given load increment $k$ the solution is obtained by cumulating the corrections computed by using equations (16) as follows

$$
\begin{gathered}
\Delta X_{k}^{i+1}=\Delta X_{k}^{i}+\delta \Delta X_{k}^{i} \\
\Delta u_{n k}^{i+1}=\Delta u_{n k}^{i}+\delta u_{n k}^{i}
\end{gathered}
$$

The tolerance of this non-linear process is applied on the variation of the crack opening corrections, i.e., $u_{i}-u_{i-1} \leq$ tolerance .

\section{Examples}

In this section one example is presented to illustrate the efficiency of the proposed numerical model to analyse crack growth. In this example, we are analysing the crack propagation in a concrete four point-bending beam proposed by Galvez et al. [9]. The analysed beam is defined in Figure 2. The geometry is given by the beam length of $675 \mathrm{~mm}$, its height of $150 \mathrm{~mm}$ and a central notch $75 \mathrm{~mm}$ deep. The material properties were taken from Galvez et al. [9], who have 
performed a laboratory test: tensile strength $f_{t}^{\prime}=3.0 \mathrm{MPa}$, Young's modulus $E=37000 \mathrm{MPa}$, Poisson ratio $v=0.20$, and the fracture energy $G_{f}=69 \mathrm{~N} / \mathrm{m}$.

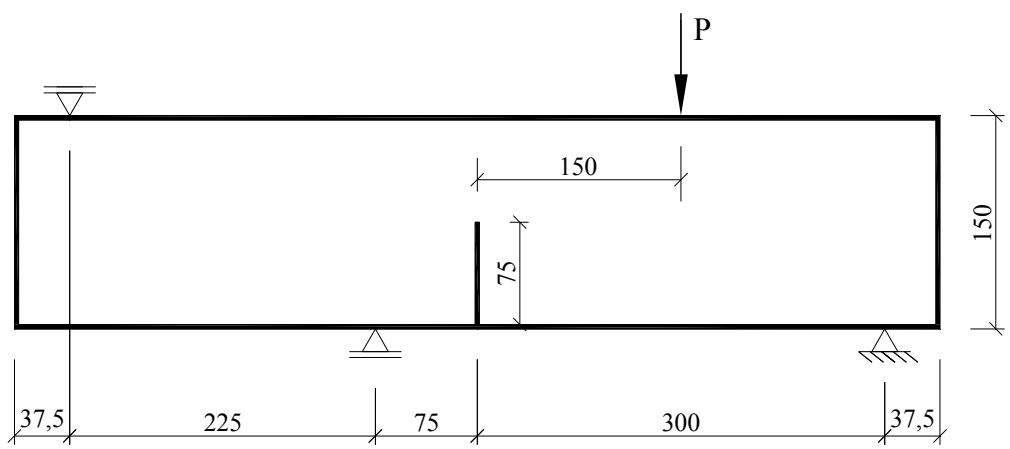

Figure 2: Analysed four-point beam. Dimensions in mm.

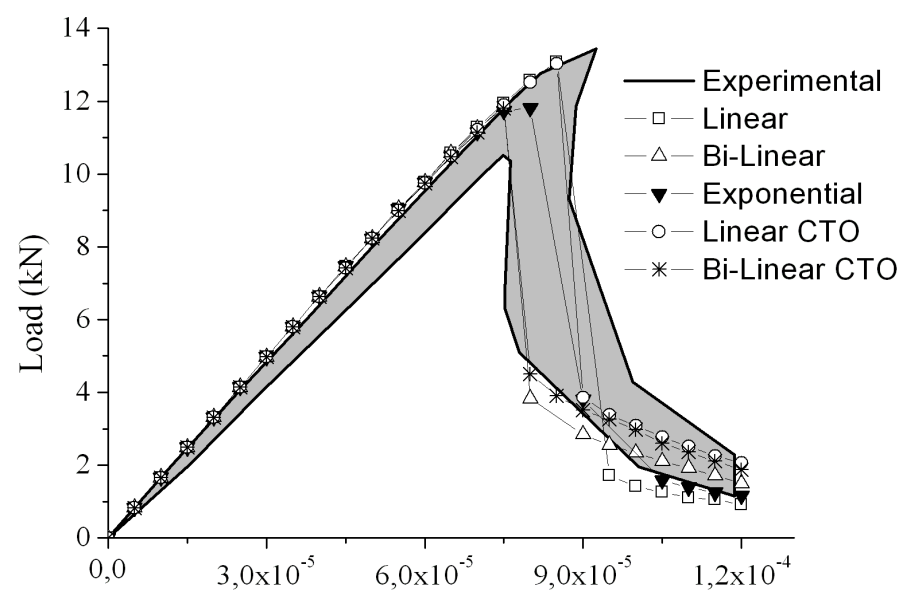

Displacement (m)

Figure 3: $\quad$ Load $\times$ displacement curves.

For the present analysis three cohesive laws were used: linear, bi-linear and exponential. For all analysed cases, the load was applied in 24 increments. The adopted tolerance within each increment was $10^{-5}$. We have tested both nonlinear system solution schemes discussed previously: a) based on the use of constant operator; b) based on the use of the consistent tangent operator. The obtained results are given in Figure 3, in which the tangent operator is identified 
by the symbol CTO. The other curves are obtained by using the constant operator. Figure 4 illustrates the crack growth process during the beam loading, leading to rupture surfaces similar than the one experimentally obtained.

Although all the results are in accordance with the experimental results, it seems that the solutions obtained by using the tangent operator is more accurate. Moreover, the tangent operator give always more stable solution requiring a reduced number of iterations at each load increment. We can see that the descendent branch obtained by using constant operator is slightly different due to the cumulated errors coming from the large number of required post-pick iterations.
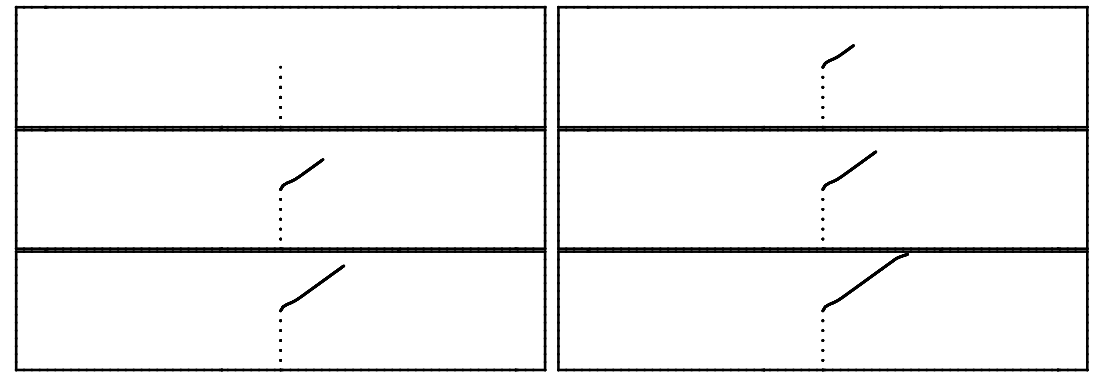

Figure 4: Crack growth profile during the load process.

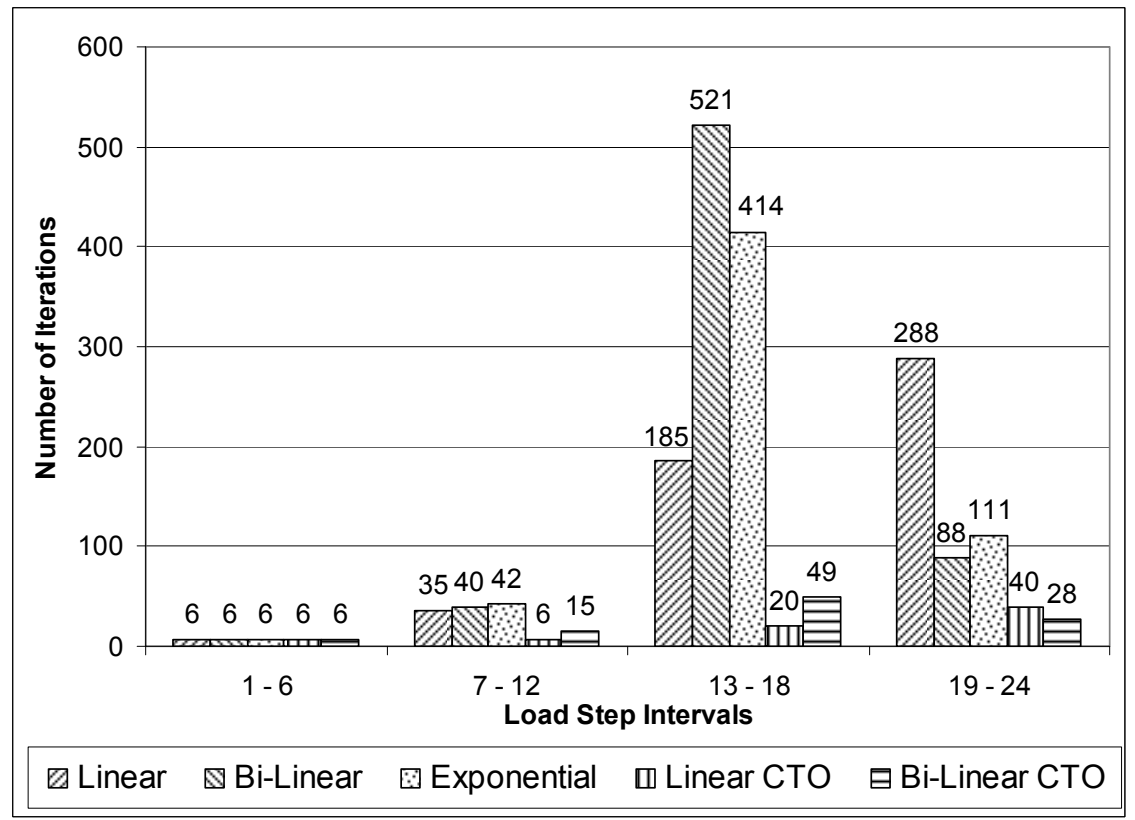

Figure 5: Number of iterations during some load increment intervals. 
To emphasize the great differences between these two system solution schemes, the iteration number to reach the equilibrium at some specific load increments are given in Figure 5. It is important to see that the constant operator scheme require very large numbers of iterations after pick what may led to more inaccurate solutions.

\section{Conclusions}

We have derived and implemented a BEM formulation to analyse cohesive crack propagation based on using consistent tangent operator. The standard procedure based on constant operator to model crack propagation has also been implemented for comparison. The formulation based on the use of tangent operator has shown to be more stable and lead to more accurate results in comparison with the standard procedure. The use of tangent operator has shown to be always recommended to analyse crack propagations, particularly for the analysis that reach the after pick region.

\section{References}

[1] Hillerborg, A., Modeer, M. \& Peterson, P.E., Analysis of crack formation and crack growth in concrete by mean of failure mechanics and finite elements. Cement Concrete Research, 6, pp. 773-782, 1976.

[2] Portela, A., Aliabadi, M.H. \& Rooke, D.P., Dual boundary element method: Efficient implementation for cracked problems. International Journal for Numerical Methods in Engineering, 33, pp. 1269-1287, 1992.

[3] Venturini, W.S., A new boundary element formulation for crack analysis. In: Brebbia, C.A., (ed.) Boundary element method XVI, Computational Mechanics Publications, Southampton and Boston, pp. 405-412, 1994.

[4] Manzoli, O.L. \& Venturini, W.S., An Implicit BEM formulation to model discontinuities in solids. Computational Mechanics, 40, pp. 901-909, 2007.

[5] Leite, L.G.S. \& Venturini, W.S., Stiff and soft thin inclusions in twodimensional solids by the boundary element method. Engineering analysis with boundary elements, 29(3), pp. 257-267, 2005.

[6] Saleh, A.L. \& Aliabadi, M.H., Crack-growth analysis in concrete using boundary-element method. Engineering Fracture Mechanics, 51 (4), pp. 533-545, 1995.

[7] Benallal, A., Fudoli, C.A. \& Venturini, W.S., An implicit BEM formulation for Gradient plasticity and localization phenomena. International Journal for Numerical Methods in Engineering, 53, pp. 1853-1869, 2002.

[8] Benallal, A., Botta, A.S. \& Venturini, W.S., On the description of localization and failure phenomena by the boundary element method. Computer Methods in Applied Mechanics and Engineering, 195, pp. 58335856, 2006.

[9] Galvez, J.C., Elices, M. Guinea, G.V. and Planas, J., Mixed mode fracture of concrete under proportional and nonproportional loading. International Journal of Fracture, 94, pp. 267-284, 1998. 